\title{
NOTES
}

\section{Characterization of Radiosensitivity of a Plastic Fiber Scintillator}

\author{
Akira TanaKa and Yuji KoJima \\ FUJISTU Ltd., 1015 Kamikodanaka, Nakahara-ku, Kawasaki 211, Japan
}

(Received August 12, 1992)

\begin{abstract}
KEY WORDS Radioactive / Scintillating Fiber / $\gamma$-Ray / Plastic / Sensor /
Dose Rate
\end{abstract}

Radiation such as $\gamma$-rays can be detected by the ionization chamber, fluorescence, and semiconductor. In nuclear power stations radiation is detected by measuring light power radiated from the scintillator using the photo-multiplier or by counting the number of the electrical pulses using a Geiger-Muller counter tube or ionization chamber. ${ }^{1}$ The scintillating fiber can be expected for sensing of $\gamma$-ray without electrical source in a part of sensing the radiation and is not affected by electromagnetic interference (EMI). In addition output power from the scintillating fiber can be transmitted to long distances from the sensing point by optical fibers without influence of EMI. So the remote control of radiation source can be simply carried out.

Recently studies of the plastic scintillating fiber doped with organic fluorescent compounds have been made and fibers having high efficiency in the conversion of $\gamma$-rays to fluorescence emmision have been obtained. ${ }^{2,3}$ These fibers have more excellent characteristics with respect to solubility in water and inorganic solution and mechanical strength, compared to the scintillating crystals such as $\mathrm{NaI}$ : Tl, CsI : $\mathrm{Tl}, \mathrm{BaF}_{2}$, and so on. This paper describes the efficiency of these plastic scintillating fibers for $\gamma$-rays of ${ }^{137} \mathrm{Cs}$ and ${ }^{60} \mathrm{Co}$.

\section{EXPERIMENTAL}

The plastic scintillating fiber consists of a polystyrene (PS) core with a refractive index of 1.59 , and a cladding of poly(methyl methacrylate) (PMMA) with a refractive index of $1.445 .^{2}$ The PS core is doped with fluorescent materials, 2-(4-tert-butylphenyl)-5-(4-biphenylyl)-1,3,4-oxadiazole, and 1,4-bis ( $p$-dimethylaminostyryl) benzene. The fluorescent color are blue. The wavelength of the maximum emission from the fiber end was $425 \mathrm{~nm}$. This fiber was coated with the black colored tube of polyethylene for protection of external light. The fiber length was $3 \mathrm{~m}$ and winded 6 turns with the diameter of $140 \mathrm{~mm}$. The fiber was arranged aganist the $\gamma$-ray source as is shown in Figure. 1.

The $\gamma$-ray sources used in the experiment were ${ }^{137} \mathrm{Cs}$ of $1 \mathrm{Ci}, 10 \mathrm{Ci}$, and $120 \mathrm{Ci}$ with

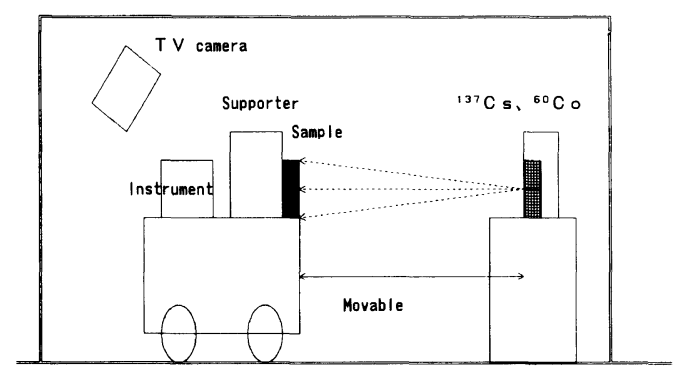

Figure 1. Schematic view of the scintillation fiber test arrangement. 
$662 \mathrm{keV}$ and ${ }^{60} \mathrm{Co}$ of $1 \mathrm{Ci}$ with $1250 \mathrm{keV}$. The dose rates for ${ }^{137} \mathrm{Cs}$ were measured as $5 \mathrm{mR} \mathrm{h}^{-1}$ to $10 \mathrm{R} \mathrm{h}^{-1}$ and those for ${ }^{60} \mathrm{Co}$ were $5 \mathrm{mRh}^{-1}$ to $100 \mathrm{mRh}^{-1}$. The irradiation conditions were based on the Japanese Industries Standard (JIS-Z4511).

The output power from the scintillating fiber was measured by an optical power meter using photodiode and a photon counting module using avalanche photodiode.

\section{RESULTS AND DISCUSSION}

\section{Principle of Scintillation}

Figure 2 shows the scintillation principle for fibers doped with scintillator. When $\gamma$-rays are incident to the fiber core and in collision with the defects such as the impurity, the end of polymer chains, and $\pi$-electronic states of PS having the condensed or linked benzenoid rings, ${ }^{4}$ the ionizing particles and electrons are emitted in the core. When these particles come into collision with the scintillator, the fluorescent rays are isotropically emitting in the core. These rays are reflected at the core-cladding interface when the incident angle, $\theta$, exceeds the critical angle, $\theta_{\mathbf{c}}\left\{\theta_{\mathbf{c}}=\right.$ $\sin ^{-1}\left(n_{2} / n_{1}\right)$, where $n_{1}$ is the core's refractive index and $n_{2}$ is the cladding's refractive index $\}$.

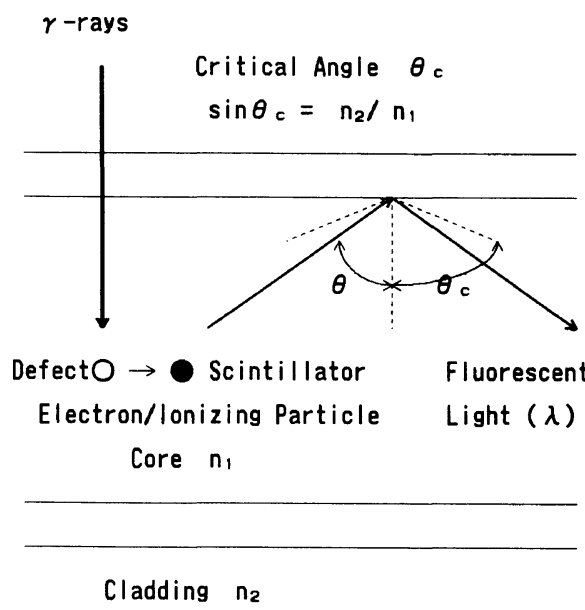

Figure 2. Optical transmission principle of scintillating fiber.
The reflected rays are transmitted along the fiber axis and output at the fiber ends.

\section{Measurement by Photon Counting Module}

Avalanche photodiode used in the photon counting module dose not generate carriers of electrons in semiconductors by the ionizing radiation X-rays, and dose not need the electrical power source with high voltage of near $500 \mathrm{Vdc}$ as in the case of photo-multiplier. Furetheremore it is not broken even if strong light power is incident upon the avalanche photodiode. Therefore this module is expected to be a more reliable and stable monitor for radiations rays compared to the photomultiplier.

Figure 3 shows the relationship between the number of counts per second from the photon counting module and dose rate of ${ }^{137} \mathrm{Cs} \gamma$-ray source irradiated the scintillating fiber. This indicates that the number of photons radiated from the scintillating fiber is detectable at above $5 \mathrm{mR} \mathrm{h}^{-1}$ and is proportional to the dose rate.

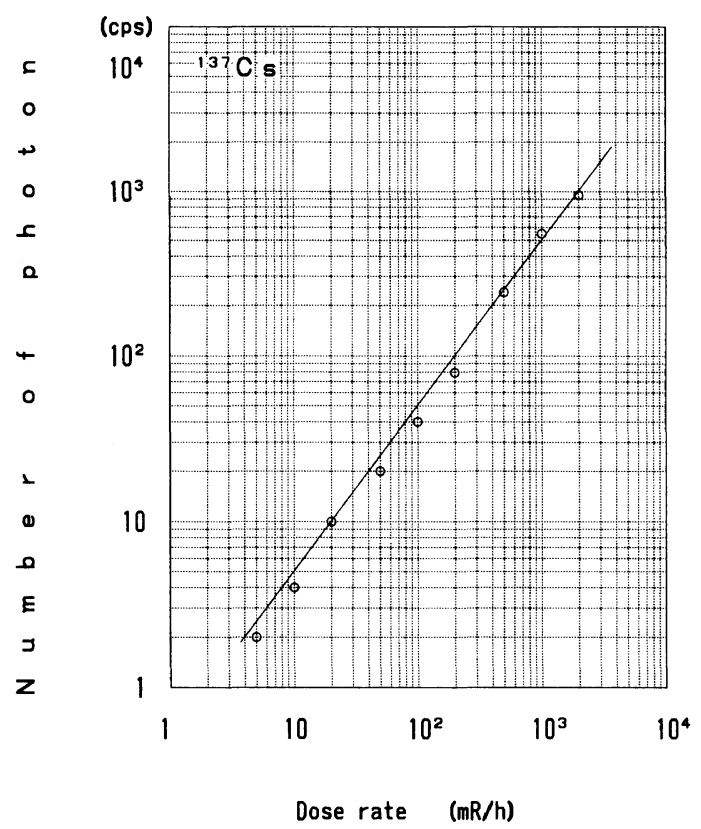

Figure 3. Number of photon counting vs. dose rate irradiated scintillating fiber with diameter of $1.0 \mathrm{~mm}$ and length of $3 \mathrm{~m}$. 


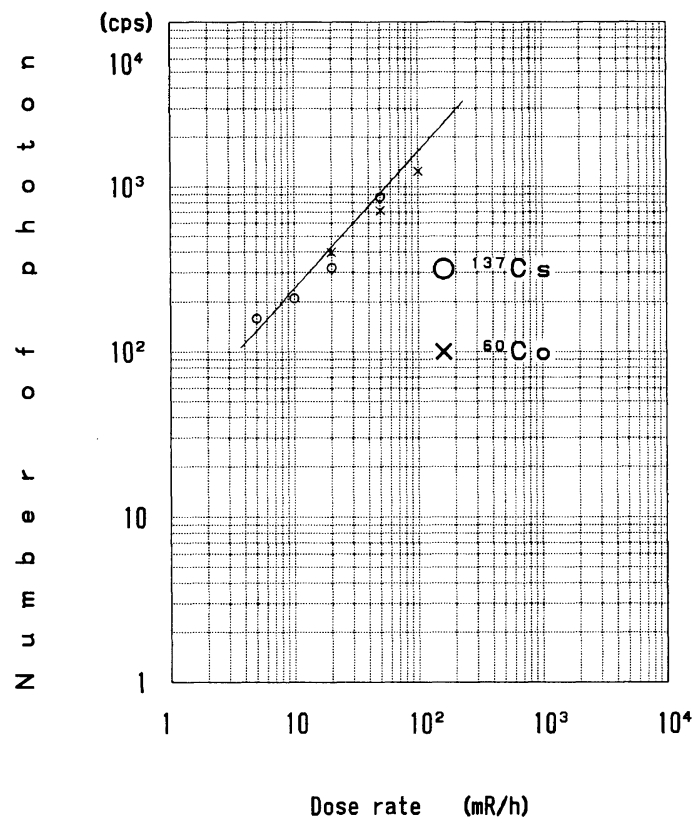

Figure 4. Photon counting number $v s$. dose rate of $\gamma$-ray sources with fiber diameter of $1.0 \mathrm{~mm}$ and length of $3 \mathrm{~m}$.

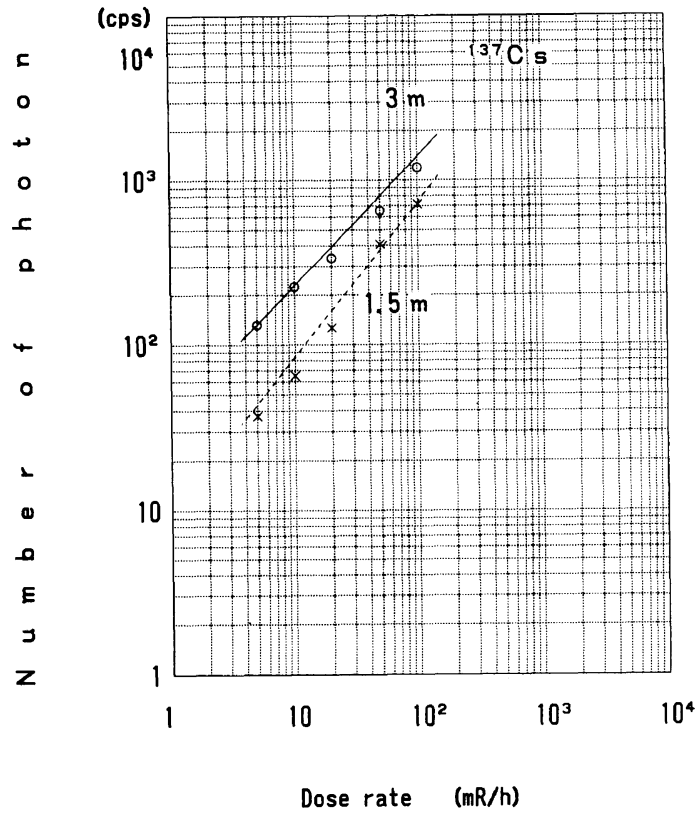

Figure 5. Photon counting number $v s$. dose rate with varying length of fiber length.

Polym. J., Vol. 25, No. 4, 1993

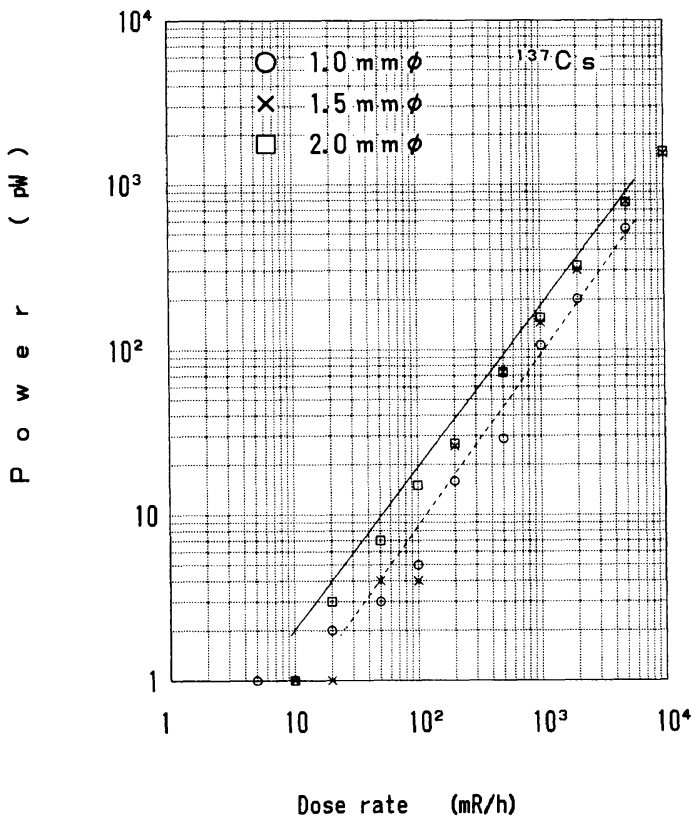

Figure 6. Output power from scintillating fiber terminal $v s$. dose rate.

Figure 4 shows the relationship between the number of counts and dose rate when the radiation sources were ${ }^{137} \mathrm{Cs}$ and ${ }^{60} \mathrm{Co}$. This suggests that the sensivity dose not depend on the $\gamma$-ray source.

Figure 5 shows the relationship between the number of counts and the dose rate when the fiber length was changed. This suggests that the fiber length affects on the apparent conversion efficiency and longer fiber length is more favorable for amplifying the number of photons.

\section{Measurement by Optical Power Meter}

Figure 6 shows the relationship between the optical output power from the scintillating fiber and the dose rate of ${ }^{137} \mathrm{Cs} \gamma$-ray source when the fiber diameter changes from $1.0 \mathrm{~mm}$ to $2.0 \mathrm{~mm}$. This also indicates that the output power is proportional to the dose rate at above $20 \mathrm{mR} \mathrm{h}^{-1}$ and longer fiber diameter results in stronger output power from the fiber terminal. However, the output power is not proportional 
to fiber diameter.

\section{CONCLUSIONS}

The plastic scintillating fiber can detect $\gamma$-rays at dose rates of above $5 \mathrm{mRh}^{-1}$ by a photoncounting module and above $20 \mathrm{mR} \mathrm{h}^{-1}$ by an optical power meter with the efficiency is about $1 \mathrm{pW}$, respectively. The measured physical values are propotional to the dose rate of $\gamma$-rays. Thus, this fiber can be expected to be a simple radiosensitive sensor based on the optical sensing techniques. Further studies to improve the conversion efficency for $\gamma$-ray to fluorescence light by the combination of other fluorescent techniques are in progress.
Acknowledgement. We acknowledge the members of nuclear instrumentation department at Fuji Electric Co., Ltd. for valuable discussion and helpful measurements.

\section{REFERENCES}

1. H. Terada, K. Sakuma, Y. Atsumi, Y. Rintz, and K. Urayama, Preprints, 1991 Annual Conference of Tohoku Section, Technical Committee for Heat and Atomic Power Plant, Sendai, Sept. 5, 1991, p 35.

2. F. Takasaki, H. Saito, T. Shimizu, S. Kondo, and O. Shinji, Nucl. Instrum. Meth., 86 (1987).

3. A. Tanaka, Y. Kojima, and H. Sawada, Polymer J., 24, 291 (1992).

4. F. D. Brooks, Nucl. Instrum. Meth., 162 (1979). 